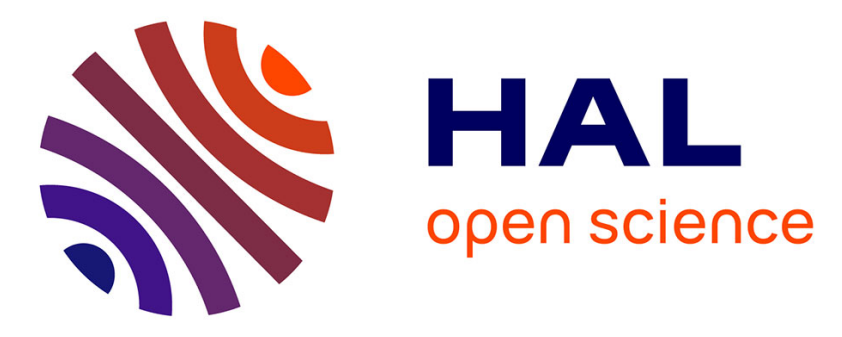

\title{
Role of resting state functional connectivity MRI in presurgical investigation of mesial temporal lobe epilepsy.
}

Gaëlle Bettus, Fabrice Bartolomei, Sylviane Confort-Gouny, Eric Guedj, Patrick Chauvel, Patrick J Cozzone, Jean-Philippe Ranjeva, Maxime Guye

\section{To cite this version:}

Gaëlle Bettus, Fabrice Bartolomei, Sylviane Confort-Gouny, Eric Guedj, Patrick Chauvel, et al.. Role of resting state functional connectivity MRI in presurgical investigation of mesial temporal lobe epilepsy.. Journal of Neurology, Neurosurgery and Psychiatry, 2010, 81 (10), pp.1147-1154. 10.1136/jnnp.2009.191460 . hal-00617799

\section{HAL Id: hal-00617799 \\ https://hal.science/hal-00617799}

Submitted on 31 Jul 2012

HAL is a multi-disciplinary open access archive for the deposit and dissemination of scientific research documents, whether they are published or not. The documents may come from teaching and research institutions in France or abroad, or from public or private research centers.
L'archive ouverte pluridisciplinaire HAL, est destinée au dépôt et à la diffusion de documents scientifiques de niveau recherche, publiés ou non, émanant des établissements d'enseignement et de recherche français ou étrangers, des laboratoires publics ou privés. 


\section{ROLE OF RESTING STATE FUNCTIONAL CONNECTIVITY MRI IN PRESURGICAL INVESTIGATION OF MESIAL TEMPORAL LOBE EPILEPSY}

Corresponding author:

Dr Maxime Guye

Address: CRMBM UMR CNRS 6612, Faculté de Médecine, 27 Boulevard Jean Moulin F 13385, Marseille cedex 05, France

Email: maxime.guye@ap-hm.fr

Phone : +33(0)491388464 Fax : +33(0)491256539

Gaelle Bettus $^{1,2,3,4}$; Fabrice Bartolomei ${ }^{2,3,4}$; Sylviane Confort-Gouny ${ }^{1,3,4}$; Eric Guedj G,3,4 $^{1,3}$

Patrick Chauvel $^{2,3,4}$; Patrick J Cozzone ${ }^{1,3,4}$; Jean-Philippe Ranjeva ${ }^{1,3,4}$; Maxime Guye ${ }^{1,3,4}$.

${ }^{1}$ Centre de Résonance Magnétique Biologique et Médicale (CRMBM), UMR CNRS 6612, Marseille, France.

2 INSERM U751, Laboratoire de Neurophysiologie et Neuropsychologie, Marseille, France.

${ }^{3}$ Faculté de Médecine, Marseille, France.

${ }^{4}$ Université de la Méditerranée Aix-Marseille II, Marseille, France.

Keywords: Functional connectivity, temporal lobe epilepsy, resting state fMRI.

\section{Word count:}

Number of words in abstract: 248

Number of words in main text: 4114

Number of figures: 2

Number of tables: 5 


\section{ABSTRACT}

Objective: We aimed at determining the ability of resting-state functional connectivity MRI (fcMRI) to lateralize/localize the epileptogenic zone in patients presenting with mesial temporal lobe epilepsy (MTLE) at the individual level.

Methods: Basal functional connectivity (BFC) was evaluated in each hemisphere of 22 MTLE patients. Two hundred volumes were acquired using a single shot GE-EPI sequence during a resting period of $10 \mathrm{~min}$ at $1.5 \mathrm{~T}$. Signal time-course was extracted from 10 regions of interest (5 ROIs in each hemisphere) usually involved in epileptogenic networks of MTLE. Normalized correlation coefficients between pairs of ROIs signal time-courses were computed to reflect BFC. Based on normative BFC values obtained from 36 controls, the number of BFC decreases and increases were determined in each hemisphere for each patient.

Results: BFC decreases were found bilaterally although the number of decreased links was significantly higher in the epileptogenic side $(\mathrm{p}=0.025)$. Conversely, $\mathrm{BFC}$ increases were found almost exclusively in the contralateral lobe leading to a strong test effect for locating the nonepileptic lobe with a sensitivity of $64 \%$ and a specificity of $91 \%(\mathrm{p}<0.001)$. The most frequently disconnected areas were the entorhinal cortex and the anterior hippocampus in the epileptic lobe, while contralateral BFC increases involved preferentially hippocampus and amygdala. Conclusions: This study demonstrates that the presence of BFC increases in the non-epileptic side was paradoxically the most specific marker of epileptogenic zone localization; and suggests that a single resting-state fcMRI could be useful in the presurgical assessment of MTLE at an individual level. 


\section{INTRODUCTION}

Since the early 90s, fMRI has been widely used as a mapping tool of brain function by measuring task-related BOLD variations. During the last decade, the neuroimaging field has witnessed a dramatic evolution of fMRI after the discovery of spontaneous fluctuations of BOLD signal at rest exhibiting coherent variations in regions belonging to the same functional networks

${ }^{1,2}$. Such coherent slow frequency fluctuations are at the basis of functional connectivity MRI (fcMRI) measurements and have contributed to bring new insights into the complex brain network organisation ${ }^{3,4}$. Moreover, the clinical impact of neurological disorders may be related to disorganization of such complex networks, justifying the need for non-invasive characterization of functional connectivity.

In mesial temporal lobe epilepsy (MTLE), using fcMRI it has already been demonstrated that cognitive impairment associated with TLE may be linked to specific cognitive network disturbances. In particular, Waites et al. showed that language impairment was related to functional connectivity decrease between regions belonging to the language network previously defined by task-related fMRI ${ }^{5}$. In addition, using fcMRI during a memory task, Addis et al. demonstrated functional disconnections of autobiographical memory network in MTLE patients with hippocampal sclerosis (HS) ${ }^{6}$. Such studies are in favour of a relationship between altered functional connectivity and cognitive dysfunctions associated with MTLE. However, although such functional networks are closely related/connected to the epileptogenic zone (EZ, i.e. responsible for seizure onset), they do not correspond to the EZ itself that mainly involves mesial temporal lobe structures. 
Nonetheless, in drug-resistant partial epilepsies, the main goal of epilepsy surgery is to locate and remove the EZ. That is why in a recent study, using resting-state fcMRI, we studied the capability of fcMRI to define functional connectivity alterations in the $\mathrm{EZ}^{7}$. In this previous study, a homogeneous group of left MTLE patients was investigated and basal functional connectivity (BFC) decreases were found within the EZ, associated with a concomitant BFC increase in contralateral lobe at the group level. However, the stringent selection criteria and the restricted number of patients did not allowed to answer the question of the clinical relevance of BFC alterations from an individual point of view which was addressed in the present work. Here, the main goal was to define the individual usefulness of resting state fcMRI in the presurgical evaluation of drug-resistant TLE. To this aim, we studied a larger and less selected (in term of handedness and epilepsy laterality) cohort of patients $(n=22)$ compared to controls $(n=36)$, and determined the sensitivity and specificity of altered connectivity as a test to lateralize/localize the epileptogenic network at the individual level. We also sought to determine individually, the extent, the types and the significance of such functional connectivity alterations.

\section{SUBJECTS/MATERIALS AND METHODS}

\section{Subjects}

Fifty-eight subjects (22 patients and 36 controls) gave their informed consent to be included in this study approved by the local Ethics Committee of Marseille Public Hospital. The 22 patients (10 with right MTLE, 5 females; and 12 with left MTLE, 7 females) were selected after a comprehensive non-invasive pre-surgical evaluation. Though exhibiting different types of pathology on conventional MRI, all other data (i.e. interictal and ictal video-EEG (especially ictal electro-clinical semiology), interictal FDG-PET, interictal +/- ictal SPECT were 
compatible with a seizure onset in the mesial temporal lobe structures. Thus, as defined in a

previous work using SEEG ${ }^{8}$. we selected these patients as they presented homogeneous electroclinical features compatible with MTLE .

Clinical characteristics of MTLE patients are reported in Table 1.

\begin{tabular}{|c|c|c|c|c|c|c|c|c|}
\hline Patient & $\operatorname{sex}$ & age & $\begin{array}{l}\text { Handed- } \\
\text { ness }\end{array}$ & $\begin{array}{c}E Z \\
\text { laterality }\end{array}$ & $\begin{array}{l}\text { Epilepsy } \\
\text { onset } \\
\text { (year) }\end{array}$ & $\begin{array}{l}\text { Seizure } \\
\text { frequency } \\
\text { (/month) }\end{array}$ & MRI visible lesion & $\begin{array}{c}\text { Seizure } \\
\text { outcome } \\
\text { (Engel's class) }\end{array}$ \\
\hline 1 & $\mathrm{M}$ & 26 & Left & Left & 13 & 4 & No visible MRI lesion & awaiting \\
\hline 2 & M & 39 & Right & Left & 33 & 6 & Left hippocampal sclerosis & I \\
\hline 3 & $\mathrm{~F}$ & 35 & Right & Left & 16 & 32 & Left hippocampal sclerosis & I \\
\hline 4 & $\mathrm{~F}$ & 23 & Right & Left & 13 & 6 & Left hippocampal sclerosis & I \\
\hline 5 & M & 40 & Right & Left & 26 & 8 & Left hippocampal sclerosis & I \\
\hline 6 & $\mathrm{~F}$ & 48 & Right & Left & 14 & 8 & Left hippocampal sclerosis & refused \\
\hline 7 & $\mathrm{~F}$ & 25 & Right & Left & 16 & 18 & Left hippocampal sclerosis & I \\
\hline 8 & $\mathrm{~F}$ & 41 & Right & Left & 27 & 6 & Left amygdala hypersignal & I \\
\hline 9 & $\mathrm{~F}$ & 25 & Right & Left & 18 & 10 & $\begin{array}{l}\text { Left parahippocampal focal } \\
\text { cortical dysplasia }\end{array}$ & III \\
\hline 10 & $\mathrm{~F}$ & 61 & Right & Left & 1 & 14 & Left hippocampal sclerosis & awaiting \\
\hline 11 & M & 37 & Right & Left & 13 & 10 & Left hippocampal dysplasia & I \\
\hline 12 & M & 20 & Right & Left & 7.5 & 4 & Left temporal pole dysplasia & II \\
\hline 13 & M & 49 & Left & Right & 18 & 18 & $\begin{array}{l}\text { Right temporal lobe } \\
\text { dysplasia }\end{array}$ & refused \\
\hline 14 & M & 57 & Left & Right & 33 & 11 & $\begin{array}{l}\text { Right post-traumatic } \\
\text { temporal lobe lesion }\end{array}$ & refused \\
\hline 15 & M & 34 & Left & Right & 30 & 12 & Right hippocampal sclerosis & I \\
\hline 16 & M & 35 & Left & Right & 15 & 1 & No visible MRI lesion & awaiting \\
\hline 17 & $\mathrm{~F}$ & 41 & Left & Right & 12 & 2 & Right hippocampal sclerosis & I \\
\hline 18 & $\mathrm{~F}$ & 24 & Right & Right & 17 & 2 & Right hippocampal sclerosis & awaiting \\
\hline 19 & M & 39 & Right & Right & 8 & 24 & $\begin{array}{c}\text { Right MTL cortical } \\
\text { dysplasia }\end{array}$ & awaiting \\
\hline 20 & $\mathrm{~F}$ & 54 & Right & Right & 13 & 16 & Right hippocampal sclerosis & I \\
\hline 21 & $\mathrm{~F}$ & 57 & Right & Right & 16 & 1.5 & Right hippocampal sclerosis & I \\
\hline 22 & $\mathrm{~F}$ & 38 & Right & Right & 12.5 & 12 & Right hippocampal sclerosis & I \\
\hline
\end{tabular}

Table 1: Clinical characteristics of MTLE patients: For patients who had surgical resection, seizure outcome is classified according to Engel's classification ${ }^{9}$ (I: Seizure free, II: rare disabling seizures ("almost seizure free"), III: worthwhile improvement, IV: no worthwhile improvement). "Awaiting" stands for patients waiting for surgery. "Refused" stands for patients who refused surgery. 
The 36 healthy controls (16 females), matched for sex $(p=0.455)$ were free of neurological disease.

In the two groups, handedness was controlled (for patients: left-handed n=6, age $=40 \pm 11$ and right-handed $n=16$, age $=38 \pm 13$; with no age differences between the two groups: Kruskal-Walis $p=0.740$; for controls left-handed $n=10$, age $=30 \pm 11$ and right-handed $n=26$, age $=29 \pm 9$; with no differences between the two groups: Kruskal-Walis $\mathrm{p}=0.791$ ).

\section{Neuropsychological testing}

Normative evaluation of memory capacity and intelligence scale of patients were assessed by the Wechsler Memory Scale - Third Edition (WMS III) (reported scores in this paper are: immediate memory quotient - IMQ, delayed memory quotient - DMQ - and working memory quotient WMQ) and the WAIS III (Wechsler Adult Intelligent Scale- Third Edition) (reported scores in this paper are: full scale intelligence quotient, verbal intelligence quotient and performance intelligence quotient). For both tests, the normal mean values were at 100 and abnormality cutoff at $85(-1.5 \mathrm{SD})$.

\section{Conventional MRI}

All subjects underwent a MRI examination (duration: $80 \mathrm{~min}$ ) in the framework of a multimodal MRI protocol on a 1.5T Magnetom Vision plus MR-scanner (Siemens, Erlangen Germany). Conventional MRI included $\mathrm{T}_{1}$-weighted images $(\mathrm{TE} / \mathrm{TR}=15 \mathrm{~ms} / 700 \mathrm{~ms}, 23$ contiguous slices, $5 \mathrm{~mm}$ slice thickness, field of view (FOV) $240 \mathrm{~mm}$, matrix 256) acquired in the AC-PC plane, $\mathrm{T}_{2^{-}}$ weighted images $(\mathrm{TE} / \mathrm{TR}=112 / 7308 \mathrm{~ms}$, FOV $240 \mathrm{~mm}$, matrix 256, 23 contiguous slices, $5 \mathrm{~mm}$ slice thickness) acquired in the bihippocampal plane, T1-weighted inversion recovery images 
$(\mathrm{TE} / \mathrm{TR}=60 / 8000 \mathrm{~ms}, \mathrm{TI}=350 \mathrm{~ms}, \mathrm{FOV}=240 \mathrm{~mm}$, matrix 512, 5mm slice thickness $)$, FLAIR images $(\mathrm{TE} / \mathrm{TR}=110 / 8000 \mathrm{~ms}, \mathrm{TI}=2500 \mathrm{~ms}, \mathrm{FOV}=240 \mathrm{~mm}$, matrix $256,5 \mathrm{~mm}$ slice thickness) acquired in a coronal axis perpendicular to the bihippocampal plane, and sagittal 3DMPRAGE images $\left(\mathrm{TE} / \mathrm{TR}=4 / 9.7 \mathrm{~ms}\right.$, isotropic voxel of $\left.1.25 \times 1.25 \times 1.25 \mathrm{~mm}^{3}\right)$.

\section{Resting state fcMRI}

Data acquisition and processing

Two hundred brain volumes (time elapsed between blocks: 4s) were acquired using a single-shot multislice gradient-echo echo-planar imaging (GE-EPI) sequence (TE 55ms, TR 4s, 30 contiguous slices, 4mm-thickness, matrix 64, FOV 256mm). Subjects were instructed to simply keep their eyes closed and to not fall asleep.

\section{Data processing}

Resting-state fcMRI acquisitions were pre-processed using SPM2 software (Welcome Institute, London, UK). After slice timing correction, images were realigned before spatial normalization (16 non linear registration 7x6x7 basis functions) and smoothing (12 mm). Sources of spurious or regionally non-specific variance related to physiological artefacts were removed by using a method previously described ${ }^{7,10,11}$. We used a regression including the CSF signal (averaged over the lateral ventricles) and the white matter signal (averaged over a region centered in the deep cerebral WM) in order to reduce the non-neuronal contributions to BOLD correlations ${ }^{7,10,}$ 11.

\section{Regions of interest}

According to the methodology described in a previous study ${ }^{7}, 10$ ROIs (5 in each hemisphere) usually involved in epileptogenic networks of mesial TLE were defined. These regions were 
automatically obtained from a digital Talairach atlas (Pick atlas toolbox, SPM2) and consisted of amygdala (Amy), entorhinal cortex (EC), anterior hippocampus (AntHip), posterior hippocampus (PostHip) and temporal pole (Brodmann area 38, BA38) ${ }^{7}$. These ROIs were used as masks applied onto the residual images to extract the mean signal time-courses from each predefined ROI.

To determine functional interactions between ROIs in each temporal lobe, correlation coefficients between pairs of signal time-courses were computed (JMP statistical software). Correlation coefficients were then normalized using the Fisher transformation $(\mathrm{rN}=0.5 \log$ $[(1+r) /(1-r)])$ to reflect BFC and to perform subsequent statistical analyses.

\section{Normative BFC values in controls}

For each control group (left and right-handed), unilateral BFC values were tested against the null hypothesis for each link (Mann Whitney test, corrected-p<0.005 (10 comparisons per group)) to determine the statistical significance of each correlation. BFC values for non-significant correlations were set to zero. Within-group effect of BFC was tested for left and right-handed controls group for each link (Wilcoxon signed rank).

For each subgroup, differences in BFC values between contralateral links were also tested (Wilcoxon rank test, corrected $\mathrm{p}<0.005$, (10 comparisons per group)).

For each hemisphere, between-group (left handed and right handed controls) comparisons of BFC values were performed for each link using a Mann Whitney test. A corrected $\mathrm{p}<0.005$ was considered to account for the 10 comparisons.

\section{BFC at individual level in patients}


In order to evaluate the added value of BFC at the individual level, z scores were calculated for patients relative to the mean and standard deviation of the corresponding subgroup of controls. To limit the potential bias related to possible variations in structural and functional asymmetries of temporal lobes, we calculated z-scores for patients relative to control group with the same handedness. Each connection from each hemisphere were classified as abnormal for $\mathrm{z}$ score values above or below 2 for each subject and the number of BFC decreases and increases were obtained for each hemisphere of each patient. In controls, this cutoff of 2 SD included the percentile 90 for each link studied and for the two groups of controls (right and left handed).

\section{Sensitivity and specificity of BFC alterations to locate the epileptogenic hemisphere}

Two types of connectivity alterations were evaluated: i) the presence of at least one link showing significant BFC decrease relative to controls in the epileptogenic temporal lobe, ii) the presence

of at least one link with significant BFC increase relative to controls in the contralateral temporal lobe.

For these two criteria, we classified BFC decreases and increases from each hemisphere of each patient as true positive, true negative, false positive and false negative. Then, we assessed: sensitivity, specificity, positive predictive value (PPV), negative predictive value (NPV), and the power of these tests using Yule's Q coefficient, $\chi^{2}$ and Yuden's index. The two criteria tested to classify the epilepsy lateralization were intentionally very simple and were defined a priori. No attempt to optimize these criteria was conducted on the present dataset to limit artificial overfitting. 


\section{Relationships between BFC and clinical data}

In patients, pair-wise correlations were performed in order to determine a potential relationship between BFC and : i) neuropsychological scores; ii) seizure frequency; and iii) disease duration. We considered that each test aimed at answering a single different question; consequently no multiple comparison corrections were applied (Spearman Rho, $\mathrm{p}<0.05$ ). 


\section{RESULTS}

\section{Neuropsychological data in patients}

Neuropsychological data are reported in Table2. Twenty one out of the 22 patients (10 with right MTLE and 11 with left MTLE) performed the whole preoperative neuropsychological evaluation.

In MTLE patients, scores for WAIS III were significantly lower than normative values for the 3 reported quotients: full scale intelligence quotient $(\mathrm{p}<0.001)$, verbal intelligence quotient $(\mathrm{p}<0.001)$ and performance intelligence quotient $(\mathrm{p}<0.001)$.

Assessment of WMSIII showed that immediate memory (IMQ) and delayed memory quotients (DMQ) were not significantly altered (respectively $\mathrm{p}=0.861$ and $\mathrm{p}=0.805$ ) and the same result was observed for the 2 subtests (auditory IMQ $p=0.206$; visual IMQ $p=0.352$; auditory DMQ $\mathrm{p}=0.494$; visual DMQ $\mathrm{p}=0.424$ ). In contrast, the working memory quotient was significantly below normative values $(\mathrm{p}<0.001)$, as well as the auditory $(\mathrm{p}=0.035)$ and visual $(\mathrm{p}<0.0001)$ working memory subtests. Variables as EZ laterality and handedness did not interfere with all quotients (WAIS III and WMS III, Wilcoxon Sign-Rank test). In addition, we did not found any significant discrepancies between visual and auditory subtests in patients $(\mathrm{p}=0.765$ in right MTLE, and $\mathrm{p}=0.680$ in left MTLE using a paired test (Wilcoxon test)). 


\begin{tabular}{|c|c|c|c|c|c|c|c|c|c|c|c|c|c|}
\hline & & \multicolumn{3}{|c|}{ WAIS III } & \multicolumn{10}{c|}{ WMS III } \\
\hline Patient & $\begin{array}{c}E Z \\
\text { laterality }\end{array}$ & $F S I Q$ & VIQ & $P I Q$ & $I M Q$ & $\begin{array}{c}\text { Visual } \\
\text { IMQ }\end{array}$ & $\begin{array}{c}\text { Aud. } \\
\text { IMQ }\end{array}$ & $D M Q$ & $\begin{array}{c}\text { Visual } \\
\text { DMQ }\end{array}$ & $\begin{array}{c}\text { Aud. } \\
\text { DMQ }\end{array}$ & WMQ & $\begin{array}{c}\text { Visual } \\
\text { WMQ }\end{array}$ & $\begin{array}{c}\text { Aud. } \\
\text { WMQ }\end{array}$ \\
\hline 1 & Left & - & - & - & - & - & - & - & - & - & - & - & - \\
\hline 2 & Left & 85 & 85 & 87 & $\mathbf{7 7}$ & $\mathbf{6 7}$ & 92 & $\mathbf{7 6}$ & $\mathbf{6 8}$ & 86 & 97 & $\mathbf{6 0}$ & 130 \\
\hline 3 & Left & $\mathbf{8 3}$ & 91 & $\mathbf{7 6}$ & 90 & 97 & 89 & $\mathbf{8 4}$ & 97 & $\mathbf{8 2}$ & $\mathbf{8 2}$ & $\mathbf{7 0}$ & $\mathbf{7 0}$ \\
\hline 4 & Left & 85 & $\mathbf{7 7}$ & 98 & 110 & 112 & 105 & 109 & 121 & 104 & 88 & 70 & 90 \\
\hline 5 & Left & 92 & 95 & 90 & 87 & 91 & $\mathbf{8 2}$ & 85 & 91 & 91 & 85 & $\mathbf{6 0}$ & 100 \\
\hline 6 & Left & 90 & 89 & 94 & 105 & 100 & 108 & 102 & 100 & 104 & 85 & $\mathbf{7 0}$ & $\mathbf{8 0}$ \\
\hline 7 & Left & 86 & 87 & 87 & 100 & 97 & 102 & 90 & 91 & 98 & $\mathbf{6 6}$ & $\mathbf{4 0}$ & $\mathbf{5 0}$ \\
\hline 8 & Left & $\mathbf{6 7}$ & $\mathbf{7 6}$ & $\mathbf{6 3}$ & 98 & 106 & 92 & 86 & 88 & 91 & $\mathbf{7 9}$ & $\mathbf{6 0}$ & $\mathbf{8 0}$ \\
\hline 9 & Left & $\mathbf{6 6}$ & $\mathbf{6 2}$ & $\mathbf{7 5}$ & 100 & 109 & 92 & 97 & 100 & 91 & $\mathbf{7 4}$ & $\mathbf{7 0}$ & $\mathbf{4 0}$ \\
\hline 10 & Left & 106 & 107 & 91 & 105 & 112 & 97 & 97 & 109 & 89 & 97 & $\mathbf{7 0}$ & 130 \\
\hline 11 & Left & 95 & 88 & 106 & 119 & 123 & 108 & 121 & 121 & 110 & $\mathbf{8 2}$ & $\mathbf{8 0}$ & $\mathbf{7 0}$ \\
\hline 12 & Left & 88 & $\mathbf{8 3}$ & $\mathbf{8 0}$ & 85 & 94 & $\mathbf{7 9}$ & $\mathbf{7 9}$ & 97 & $\mathbf{8 4}$ & 91 & $\mathbf{7 0}$ & 100 \\
\hline 13 & Right & 92 & 93 & 91 & 98 & 100 & 97 & 106 & 109 & 104 & 88 & $\mathbf{8 0}$ & $\mathbf{8 0}$ \\
\hline 14 & Right & 106 & 104 & 108 & 119 & 123 & 108 & 130 & 121 & 123 & 114 & 120 & 130 \\
\hline 15 & Right & 96 & 94 & 100 & 125 & 129 & 111 & 130 & 127 & 114 & 97 & $\mathbf{7 0}$ & $\mathbf{7 0}$ \\
\hline 16 & Right & 89 & 86 & 96 & 100 & 109 & 92 & 106 & 121 & 101 & 100 & 110 & 90 \\
\hline 17 & Right & $\mathbf{7 9}$ & 88 & $\mathbf{7 3}$ & 108 & 115 & 99 & 111 & 118 & 110 & 88 & $\mathbf{6 0}$ & 100 \\
\hline 18 & Right & 87 & 94 & $\mathbf{8 0}$ & 107 & 110 & 111 & 111 & 112 & 119 & 105 & $\mathbf{8 0}$ & 140 \\
\hline 19 & Right & $\mathbf{6 4}$ & $\mathbf{6 3}$ & 99 & $\mathbf{6 3}$ & $\mathbf{6 1}$ & $\mathbf{7 0}$ & $\mathbf{6 7}$ & $\mathbf{6 4}$ & $\mathbf{7 9}$ & $\mathbf{7 9}$ & $\mathbf{7 0}$ & $\mathbf{6 0}$ \\
\hline 20 & Right & $\mathbf{5 8}$ & $\mathbf{6 2}$ & $\mathbf{5 5}$ & $\mathbf{8 0}$ & 94 & $\mathbf{7 2}$ & $\mathbf{7 8}$ & 94 & $\mathbf{7 9}$ & $\mathbf{7 7}$ & $\mathbf{4 0}$ & $\mathbf{6 0}$ \\
\hline 21 & Right & 86 & 86 & $\mathbf{8 1}$ & 103 & 103 & 102 & 96 & 106 & 96 & $\mathbf{7 7}$ & $\mathbf{6 0}$ & $\mathbf{6 0}$ \\
\hline 22 & Right & 92 & 89 & 98 & 110 & 112 & 105 & 104 & 100 & 98 & $\mathbf{7 9}$ & $\mathbf{6 0}$ & $\mathbf{8 0}$ \\
\hline
\end{tabular}

Table 2: Neuropsychological characteristics of MTLE patients: Evaluation of intelligence scale and memory capacities by the Wechsler adult intelligent scale - Third Edition (WAIS III) (reported scores: full scale intelligence quotient (FSIQ), verbal intelligence quotient (VIQ) and performance intelligence quotient (PIQ)) and the Wechsler memory scale - Third Edition (WMS III) (reported scores: immediate memory quotient (IMQ), delayed memory quotient (DMQ) and working memory quotient (WMQ), all with visual and auditory quotients). Scores in bold correspond to abnormal performances (under 1.5 SD; normal mean $=100$ ).

\section{Normative BFC in controls}

In right-handed controls, significant BFC was present bilaterally for all the possible links $(\mathrm{p}<0.05)$ except for the link EC-PostHip in the two hemispheres. Left-right asymmetry in BFC was present, with a left predominance for the links AntHip-PostHip, Amy-AntHip and AmyPostHip, while a right predominance was observed for the link Amy-EC ( $\mathrm{p}<0.005)$. 
The same significant links were observed in left handed and right handed controls. Left-right asymmetry in BFC was present with a left predominance for the link Amy-AntHip, while a right predominance was observed for the link Amy-EC ( $<<0.005)$.

Relative to the left-handed group, the right-handed controls showed BFC increase in the left temporal lobe for the links Amy-PostHip and AntHip-PostHip (p<0.005) (Figure 1). Group data are reported in Table 3).

Accordingly, individual results in patients were compared to the subgroup of controls with the same handedness.

\begin{tabular}{|c|c|c|c|c|}
\hline \multirow{3}{*}{ ROls } & \multicolumn{2}{|c|}{ Right handed } & \multicolumn{2}{|c|}{ Left handed } \\
\hline & Right lobe & Left lobe & Right lobe & Left lobe \\
\hline & mean & mean & mean & mean \\
\hline Amy - BA38 & $\begin{array}{c}0.329 \\
(0.154)\end{array}$ & $\begin{array}{c}0.339 \\
(0.141)\end{array}$ & $\begin{array}{c}0.329 \\
(0.198)\end{array}$ & $\begin{array}{c}0.340 \\
(0.139)\end{array}$ \\
\hline Amy - EC & 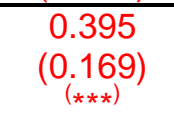 & 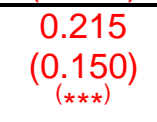 & $\begin{array}{c}0.378 \\
(0.126)\end{array}$ & $\begin{array}{c}0.240 \\
(0.118)\end{array}$ \\
\hline Amy - AntHip & $\begin{array}{c}0.363 \\
(0.155)\end{array}$ & $\begin{array}{c}0.475 \\
(0.128)\end{array}$ & $\begin{array}{c}0.347 \\
(0.168)\end{array}$ & $\begin{array}{c}0.480 \\
(0.091)\end{array}$ \\
\hline Amy - PostHip & 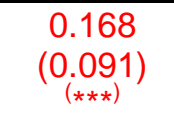 & 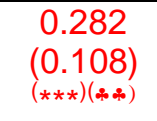 & $\begin{array}{c}0.106 \\
(0.096)\end{array}$ & $\begin{array}{c}0.120 \\
(0.131) \\
(\neq * \infty)\end{array}$ \\
\hline BA38 - EC & $\begin{array}{c}0.250 \\
(0.182)\end{array}$ & $\begin{array}{c}0.268 \\
(0.159)\end{array}$ & $\begin{array}{c}0.228 \\
(0.165)\end{array}$ & $\begin{array}{c}0.300 \\
(0.089)\end{array}$ \\
\hline BA38 - AntHip & $\begin{array}{c}0.296 \\
(0.133) \\
\end{array}$ & $\begin{array}{c}0.288 \\
(0.160) \\
\end{array}$ & $\begin{array}{c}0.351 \\
(0.106) \\
\end{array}$ & $\begin{array}{c}0.320 \\
(0.121) \\
\end{array}$ \\
\hline BA38 - PostHip & $\begin{array}{c}0.175 \\
(0.113)\end{array}$ & $\begin{array}{c}0.214 \\
(0.120)\end{array}$ & $\begin{array}{c}0.174 \\
(0.100)\end{array}$ & $\begin{array}{c}0.180 \\
(0.145)\end{array}$ \\
\hline EC - AntHip & $\begin{array}{c}0.208 \\
(0.117)\end{array}$ & $\begin{array}{c}0.138 \\
(0.141)\end{array}$ & $\begin{array}{c}0.132 \\
(0.163)\end{array}$ & $\begin{array}{c}0.160 \\
(0.115)\end{array}$ \\
\hline EC - PostHip & ns & ns & ns & ns \\
\hline AntHip - PostHip & $\begin{array}{l}0.310 \\
(0.07)\end{array}$ & $\begin{array}{c}0.418 \\
(0.133) \\
(\infty+\infty)\end{array}$ & $\begin{array}{c}0.284 \\
(0.112)\end{array}$ & $\begin{array}{c}0.250 \\
(0.155) \\
(\omega *)\end{array}$ \\
\hline
\end{tabular}

Table 3: Normalized correlation coefficients (and standard deviation) between ROIs in each lobe, for right-handed and left-handed controls. Significant left-right lateralization within one group of control (willcoxon rank test) $* \mathrm{p}<0.05 ; * * \mathrm{p}<0.01 ; * * * \mathrm{p}<0.001$. Significant left handed and right handed controls differences (Mann Whitney U-test) ${ }^{*} p<0.05, \cdots p<0.01,{ }^{* *} p<0.001$. ns: no significant link. 


\section{Altered BFC in patients}

Individual data are reported in Table 4.

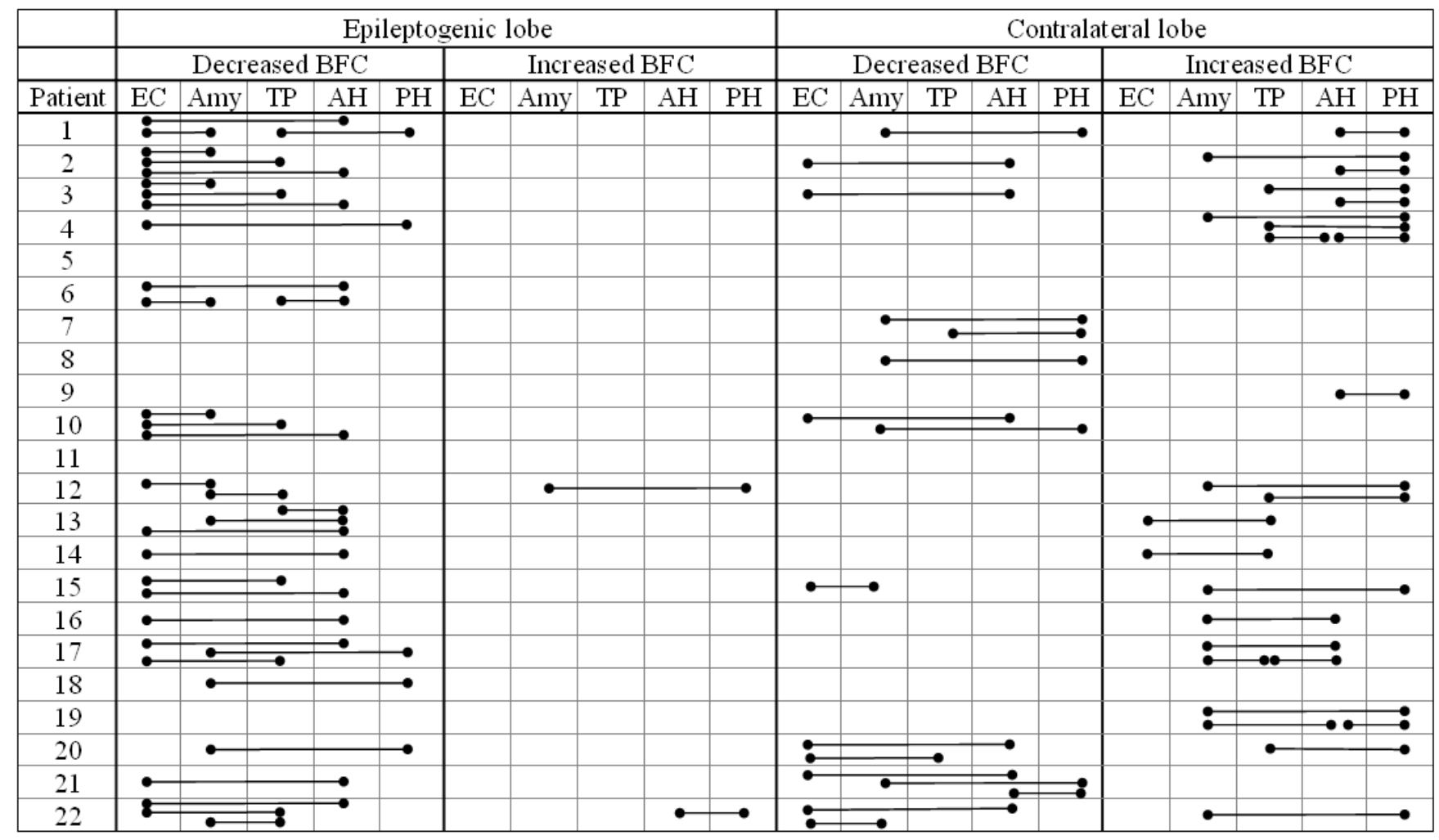

Table 4: Bilateral regional occurrence of abnormal pair-wise basal functional connectivity in MTLE patients: Columns correspond to decreased and increased links in patients, relative to controls (above or below 2 standard deviations) in the epileptogenic zone (EZ) and in the contralateral lobe and for each pair of region of interest. Used abbreviations: BFC: Basal Functional Connectivity; EC: Entorhinal Cortex; Amy: Amygdala; TP: Temporal Pole (BA38); AH: Anterior Hippocampus; PH: Posterior Hippocampus.

Two out of the 22 MTLE patients showed a normal BFC profile (patients 5 and 11). BFC

decreases were observed in 18 out of the 22 patients (patients 1-4, 6-8, 10, 12-18, 20-22). Sixteen had at least one link BFC decrease in the EZ (patients 1-4, 6, 10, 12-18, 20-22) while 10 had a contralateral BFC decrease (patients 1-3, 7, 8, 10, 15, 20-22). Eight had bilateral BFC decreases 
(patients 1-3, 10, 15, 20-22), 8 had unilateral BFC decrease(s) in the EZ (patients 4, 6, 12, 13, 14, 16-18) and only 2 had unilateral BFC decrease(s) in the contralateral hemisphere (patients 7, 8). Fourteen out of the 22 patients had BFC increases (patients 1-4, 9, 12-17, 19, 20, 22). Twelve had unilateral BFC increase(s) restricted to the contralateral temporal lobe (patients 1-4, 9, 13$17,19,20)$ while only 2 had a BFC increase in the two temporal lobes (patients 12, 22). Note that in these two patients the number of links with BFC increase was the same or higher in the lobe contralateral to seizure.

At the group level, the number of links with BFC decrease was higher in the epileptogenic side $($ mean $=1.545, \mathrm{SD}=1.262)$ compared to the contralateral hemisphere $($ mean $=0.727, \mathrm{SD}=0.935)$ $(\mathrm{p}=0.025)$ (Figure $2 \mathrm{a})$. Moreover, the number of links with BFC increase was higher in the nonepileptogenic temporal lobe (mean=1.091, $\mathrm{SD}=1.192$ ) in comparison with the epileptogenic side $($ mean $=0.091, \mathrm{SD}=0.294)(\mathrm{p}<0.001),($ Figure $2 \mathrm{~b})$.

\section{Qualitative study of the most altered areas}

The most disconnected structure of the epiletogenic network studied was the EC. Indeed, in the whole group of patients, we observed 25 links with significant BFC decreases involving EC in the EZ (36.76\% of links with BFC decreases). The second most altered area was the AntHipp $(16 ; 23.53 \%)$.

In the contralateral temporal lobe, BFC increases most often involved PostHipp (16; 33.60\%), AntHipp (11;22.91\%) and Amy $(10 ; 20.83 \%)$.

\section{Diagnostic value of BFC to lateralize epilepsy (Table 5)}

Table 5 shows the sensitivity, specificity, PPV and NPV for each test. 
BFC decreases were found bilaterally exhibiting a lateralization power with a sensitivity of $\sim 73 \%$ and a specificity of $54.5 \%$. Conversely, BFC increases were found almost exclusively in the contralateral lobe leading to a strong test effect for locating the non- epileptic lobe with a sensitivity of $64 \%$ and a specificity of $91 \%(\mathrm{p}<0.001)$.

\begin{tabular}{|c|c|c|c|c|}
\hline \multirow{5}{*}{$\begin{array}{c}\text { Diagnostic test: } \\
\text { ability to localize } \\
\text { the epileptogenic } \\
\text { side. }\end{array}$} & & Left TLE & Right TLE & All \\
\hline & True positive & 7 & 9 & 16 \\
\hline & False negative & 5 & 1 & 6 \\
\hline & False positive & 6 & 4 & 10 \\
\hline & True negative & 6 & 6 & 12 \\
\hline \multirow{7}{*}{$\begin{array}{l}\text { Marker tested: } \\
\text { presence of at } \\
\text { least } 1 \text { BFC } \\
\text { decrease }\end{array}$} & sensitivity & $58.33 \%$ & $90 \%$ & $72.73 \%$ \\
\hline & specificity & $50 \%$ & $60 \%$ & $54.55 \%$ \\
\hline & PPV & $53.85 \%$ & $69.23 \%$ & $61.54 \%$ \\
\hline & NPV & $54.55 \%$ & $85.71 \%$ & $66.67 \%$ \\
\hline & Yuden's index & 0.08 & 0.5 & 0.27 \\
\hline & Yules's Q & 0.17 & 0.86 & 0.52 \\
\hline & $\mathrm{p}\left(\chi^{2}\right)$ & $>0.05$ & $<0.02$ & $>0.05$ \\
\hline \multicolumn{5}{|l|}{ b) } \\
\hline \multirow{6}{*}{$\begin{array}{l}\text { Diagnostic test: } \\
\text { ability to localize } \\
\text { the non- } \\
\text { epileptogenic side }\end{array}$} & & $\overline{\text { Left TLE }}$ & Right TLE & All \\
\hline & True positive & 6 & 8 & 14 \\
\hline & False negative & 6 & 2 & 8 \\
\hline & False positive & 1 & 1 & 2 \\
\hline & True negative & 11 & 9 & 20 \\
\hline & sensitivity & $50 \%$ & $80 \%$ & $63.64 \%$ \\
\hline \multirow{6}{*}{$\begin{array}{c}\text { Marker tested: } \\
\text { presence of at } \\
\text { least } 1 \text { BFC } \\
\text { increase }\end{array}$} & specificity & $91.67 \%$ & $90 \%$ & $90.91 \%$ \\
\hline & PPV & $85.71 \%$ & $88.89 \%$ & $87.8 \%$ \\
\hline & NPV & $64.71 \%$ & $81.82 \%$ & $71.43 \%$ \\
\hline & Yuden's index & 0.42 & 0.7 & 0.55 \\
\hline & Yules's Q & 0.83 & 0.95 & 0.89 \\
\hline & $\mathrm{p}\left(\chi^{2}\right)$ & $<0.05$ & $<0.01$ & $<0.001$ \\
\hline
\end{tabular}

Table 5: Sensitivity and specificity of basal functional connectivity (BFC) alterations to lateralize the epileptogenic zone: a) Diagnostic power of BFC decreases detection to lateralize the epileptogenic zone (EZ). True positive: patients with at least one decreased link in the EZ lobe. False negative: patients with no decreased link in the EZ lobe. False positive: patients with at least one decreased link in the contralateral lobe. True negative: patients with no decreased link in the contralateral lobe. PPV: Positive Predictive Value; NPV: Negative Predictive Value.

b) Diagnostic power of the presence of at least one abnormal link showing increased basal functional connectivity to localize the temporal lobe contralateral to EZ. True positive: patients with at least one increased link in the contralateral lobe to EZ. False negative: patients with no increased link in the contralateral lobe to EZ. False positive: patients with at least one increased 
link in the EZ lobe. True negative: patients with no increased link in the EZ lobe. PPV: Positive Predictive Value; NPV: Negative Predictive Value.

\section{Relationship between BFC and visible lesions on conventional MRI}

Thirteen patients had unilateral HS; 4 had cortical dysplasia and 2 had other types of lesions (1 temporal post-traumatic lesion and 1 hippocampal dysplasia 1). We tested (Wilcoxon rank test) the influence of MRI visible lesion on neuropsychological data and the number of altered links, by comparing data from the subgroup of 13 patients with HS and the subgroup of 9 patients with no HS. No difference was observed for neuropsychological data between HS and no HS subgroup. In addition no significant difference was found between the two groups concerning the number of altered BFC, neither the number of BFC decreases in the EZ lobe nor the number of BFC increases in the contralateral lobe.

\section{Relationships between BFC and clinical data}

No significant statistical relationship was observed between seizure frequency, disease duration and BFC. However, correlating BFC values with neuropsychological memory scores in patients, we observed a significant positive correlation between working memory (WM) scores and increased BFC in the non- epileptogenic temporal lobe. Indeed, the Amy-BA38 link, contralateral to EZ, was correlated with working memory quotient (WMQ) (Spearman Rank test, $\mathrm{p}=0.0494)$ and auditory WMQ ( $\mathrm{p}=0.0413)$. In addition, the EC-AntHipp link contralateral to EZ, was positively correlated with WMQ ( $\mathrm{p}=0.0109)$, auditory WMQ ( $\mathrm{p}=0.0043)$ and visual WMQ $(\mathrm{p}=0.0024)$. We also tested the spread between verbal and visual performance scores as a potential parameter. However, we did not found any significant discrepancies between visual and 
auditory subtests in patients ( $\mathrm{p}=0.765$ in right sided MTLE, and $\mathrm{p}=0.680$ in left sided MTLE using a paired test (Wilcoxon test)).

\section{DISCUSSION}

Using resting state fcMRI we studied BFC in patients presenting with MTLE in order to test the accuracy of the method in the EZ location.

In this work, the main results were: (i) presence of a BFC alteration pattern characterized by a bilateral decrease predominant in the epileptic side, and an unilateral increase almost exclusively observed in the contralateral "non-epileptic" lobe, (ii) evidence that the most frequently disconnected areas were the entorhinal cortex and the anterior hippocampus in the epileptic lobe, while contralateral BFC increases involved preferentially posterior hippocampus, anterior hippocampus and amygdala, (iii) at the individual level, presence of BFC increases in the nonepileptic (contralateral) side was paradoxically the most specific marker of EZ localization .

\section{Basal functional MTL connectivity lateralization depending on handedness}

In right-handed controls, an asymmetrical leftward organization of BFC was present between MTL structures. These results were discussed in a previous study ${ }^{7}$ in line with structural data obtained by quantitative diffusion tensor tractography ${ }^{12}$. Compared to right-handed controls, left-handed controls BFC was less leftward lateralized, except for amygdala-anterior hippocampus link. This interesting finding clearly showed an effect of handedness on BFC in controls. Effect of handedness on temporal lobes volumes has already been reported in studies using morphometry ${ }^{13}$. However, to our knowledge, no study has investigated handedness effect on BFC, and resting-state connectivity studies include preferentially right-handed subjects 
probably in order to control the handedness variable ${ }^{14}$. Here we demonstrate that asymmetries in brain lobes due to handedness are not only anatomical but also functional. According to this impact of handedness on BFC in controls, we controlled this variable by comparing individual results in patients to the subgroup of controls with the same handedness. However, the fact that patients might have handedness changes due to pathological plasticity must also be acknowledged.

\section{Basal functional connectivity decreases in MTLE is not specific of EZ}

In patients, at an individual level, BFC decreases were often bilateral even if the epileptic side was preferentially affected. Therefore, not surprisingly, we found a low specificity and sensitivity of BFC decreases as a diagnostic test for EZ localization. This is concordant with other imaging studies concerning structural as well as metabolic data. Bilateral structural abnormalities have already been shown using morphometry and diffusion weighted imaging even in patients presenting with visible unilateral HS ${ }^{15-20}$. In addition, bilateral metabolic abnormalities were also measured by magnetic resonance spectroscopy and positron emission tomography with fluoro-desoxyglucose in mesial temporal structures ${ }^{21,22}$. Such abnormalities may be observed in the presence or absence of HS and may involve areas devoid of any visible lesions ${ }^{16}$. The concordance between BFC and these metabolic abnormalities which have been demonstrated to be linked to interictal epileptiform discharges (IED), suggests a potential relationship between BFC and spikes ${ }^{21,23}$.

The most involved areas in these alterations were the EC and AntHip. These two structures have a crucial role in the epileptogenic network of TLE ${ }^{16,24-26}$. This has been demonstrated by their participation to the dynamics of seizure onset using depth recording, and also by the atrophy that 
preferentially affects these structures in MTLE patients. EC and hippocampus interactions have been well known for decades, EC being the privileged relay of the afferent pathways to the hippocampus from neocortical areas ${ }^{27-29}$. Finally, a quantified study of epileptogenicity based on the measure of fast EEG discharges at seizure onset has recently shown that these two structures are the most epileptogenic in patients with MTLE associated with normal MRI or HS ${ }^{25}$. BFC decreases are concordant with a recent structural connectivity study conducted in a very large group of TLE patients, demonstrating bilateral decreased connectivity between the EC and the other mesial temporal structures as well as the neocortex ${ }^{16}$. Such functional and structural connectivity alterations are likely to be linked to plasticity secondary to both generation and propagation of repetitive epileptiform discharges ${ }^{30-32}$.

It is noteworthy that the results of resting state fcMRI studies apparently contradict those based on EEG connectivity. Indeed, an increase in functional connectivity in the EZ has been measured from intracranial recordings ${ }^{33,34}$. The mechanisms underlying these discrepancies are unknown to date and require further investigations. However, one of the main hypotheses remains a neurovascular decoupling in epileptic patients ${ }^{34}$. Another hypothesis is that EEG connectivity and fcMRI capture different phenomena occurring on different time scale. Indeed, fcMRI is based on the cross-correlations between very slow fluctuations signals $(<0.1 \mathrm{~Hz})$ whereas EEG connectivity considers a broader frequency band but usually above $0.5 \mathrm{~Hz}$ (and up to 100-200 $\mathrm{Hz})^{2,33,34}$. This may suggest complementary information from both modalities rather than a real discrepancy. Thus, in epileptic patients functional connectivity based on slow fluctuations could be rather linked to the actual functional integrity of the macrosystems studied ${ }^{6,14}$ whereas the one based on faster fluctuations could be rather linked to abnormally high connectivity secondary to pathological electrophysiological changes ${ }^{33-35}$. 


\section{Increased basal functional connectivity is specific to the contralateral lobe to $\mathrm{EZ}$}

Because BFC increase is almost exclusively localized in the contralateral lobe to EZ, its detection permits indirect localization of the EZ with a very high specificity. Moreover, BFC increases involved more specifically PostHip, AntHip and Amy. Increased functional connectivity of the hippocampus and Amy has already been described in patients with Alzheimer's disease compared to controls during a delayed match-to-sample face recognition task $^{36}$. This increased connectivity was interpreted as reflecting an implicit signalling of emotional content to increase memory capacities. Although our resting state protocol did not allow assessment of connectivity behaviour specifically during this type of task, we hypothesise that BFC increases may reflect the same kind of compensatory mechanisms. In addition it is now accepted that MTL not only plays a crucial role in declarative memory ${ }^{37,38}$, but is also implicated in $\mathrm{WM}^{39-42}$. Altered WMQ in patients corroborate these findings. We observed altered WMQ in both left and right MTLE patients, and for both visual and auditory subtests. Moreover, the significant correlations between WMQ and increased BFC also support the hypothesis of MTL involvement in WM processes. However, our study was focused on an $a$ priori network involving only the MTL structures and did not include extra-temporal regions for statistical considerations (limited number of ROIs for multiple comparisons). It is thus possible that alterations of WMQ were also linked to wider functional network dysfunctions such as those involving frontal lobe. Accordingly, Bernhardt et al. found an increased structural connectivity between EC and homolateral orbitofrontal areas suggesting other compensatory mechanisms involving extra-temporal areas ${ }^{16}$. In addition WMQ is a sensitive test frequently affected in 
neurological disorders despite its lack of specificity ${ }^{43}$. This could also explain why, in this context, this cognitive test is found to correlate with BFC increases.

\section{Diagnostic power of BFC to localize EZ}

This study mainly shows that fcMRI is not able to directly determine the regions involved in seizure generation, since bilateral decrease in temporal lobe structures is the rule. However, BFC increases may be a reliable indicator of functional changes in the region contralateral to the EZ. Currently, several studies have reported fcMRI data in drug-resistant partial epilepsies ${ }^{20,44,45}$. However, to our knowledge, none have concentrated on individual results and on the potential clinical usefulness of the technique in the presurgical assessment of these epilepsies. The advantage of our study is that we were able to determine with a specificity of about $91 \%$ the EZ lateralization. This method could represent an interesting clinical tool to help physicians. We acknowledge that the majority of patients presented with HS which is already a robust feature for EZ lateralization. However, contralateral increased BFC was also found in 7 of the 9 patients with no HS. Our findings suggest that such BFC alterations represent a functional plasticity secondary to epileptic processes independent of the causal lesion. This is in accordance with the structural connectivity study by Bernhardt et al. which found no significant relationship between TLE-related atrophy and EC connectivity ${ }^{16}$. In addition, patients presenting with normal-appearing MRI also exhibited such contralateral increased BFC. Thus, we propose that resting-state fcMRI is a useful technique that could be added to the presurgical assessment of drug-resistant partial epilepsies. 
Acknowledgement: The authors thank Virginie Laguitton for the neuropsychological assessment of patients, and Dr. Aileen McGonigal for the revision of the English version. Fundings: This work was supported by CNRS, INSERM and ANR (CONNECTEPI). GB is the recipient of a PhD research grant delivered by the Region 'Provence Alpes-Côte d'Azur' and Deltamed.

Competing interests: None

Ethics approval: This study approved by the local Ethics Committee of Marseille Public Hospital. There is no identifiable patient information in the manuscript.

Licence for Publication: The Corresponding Author has the right to grant on behalf of all authors and does grant on behalf of all authors, an exclusive licence (or non exclusive for government employees) on a worldwide basis to the BMJ Publishing Group Ltd to permit this article (if accepted) to be published in JNNP and any other BMJPGL products and sublicences such use and exploit all subsidiary rights, as set out in our licence. 


\section{REFERENCES}

1. Lowe MJ, Mock BJ, Sorenson JA. Functional connectivity in single and multislice echoplanar imaging using resting-state fluctuations. Neuroimage 1998;7:119-32.

2. Fox MD, Raichle ME. Spontaneous fluctuations in brain activity observed with functional magnetic resonance imaging. Nat Rev Neurosci 2007;8:700-11.

3. Van Geit W, Achard P, De Schutter E. Neurofitter: a parameter tuning package for a wide range of electrophysiological neuron models. Front Neuroinformatics 2007;1:1.

4. Guye M, Bartolomei F, Ranjeva JP. Imaging structural and functional connectivity: towards a unified definition of human brain organization? Curr Opin Neurol 2008;21:393-403.

5. Waites AB, Briellmann RS, Saling MM, Abbott DF, Jackson GD. Functional connectivity networks are disrupted in left temporal lobe epilepsy. Ann Neurol 2006;59:335-43.

6. Addis DR, Moscovitch M, McAndrews MP. Consequences of hippocampal damage across the autobiographical memory network in left temporal lobe epilepsy. Brain 2007;130:2327-42.

7. Bettus G, Guedj E, Joyeux F, et al. Decreased basal fMRI functional connectivity in epileptogenic networks and contralateral compensatory mechanisms. Hum Brain Mapp 2009;30:1580-91.

8. Maillard L, Vignal JP, Gavaret M, et al. Semiologic and electrophysiologic correlations in temporal lobe seizure subtypes. Epilepsia 2004;45:1590-9.

9. Engel J VP, Rasmussen T, Ojemann L. Outcome with respect to epileptic seizures. In: Engel J, ed. Surgical Treatment of the Epilepsies 1993:609-622.

10. Bartels A, Zeki S. The chronoarchitecture of the cerebral cortex. Philos Trans R Soc Lond B Biol Sci 2005;360:733-50. 
11. Vincent JL, Snyder AZ, Fox MD, et al. Coherent spontaneous activity identifies a hippocampal-parietal memory network. J Neurophysiol 2006;96:3517-31.

12. Nucifora PG, Verma R, Melhem ER, Gur RE, Gur RC. Leftward asymmetry in relative fiber density of the arcuate fasciculus. Neuroreport 2005;16:791-4.

13. Herve PY, Crivello F, Perchey G, Mazoyer B, Tzourio-Mazoyer N. Handedness and cerebral anatomical asymmetries in young adult males. Neuroimage 2006;29:1066-79.

14. Waites AB, Stanislavsky A, Abbott DF, Jackson GD. Effect of prior cognitive state on resting state networks measured with functional connectivity. Hum Brain Mapp 2005;24:59-68.

15. Sankar T, Bernasconi N, Kim H, Bernasconi A. Temporal lobe epilepsy: differential pattern of damage in temporopolar cortex and white matter. Hum Brain Mapp 2008;29:931-44.

16. Bernhardt BC, Worsley $\mathrm{KJ}$, Besson $\mathrm{P}$, et al. Mapping limbic network organization in temporal lobe epilepsy using morphometric correlations: insights on the relation between mesiotemporal connectivity and cortical atrophy. Neuroimage 2008;42:515-24.

17. Seidenberg M, Kelly KG, Parrish J, et al. Ipsilateral and contralateral MRI volumetric abnormalities in chronic unilateral temporal lobe epilepsy and their clinical correlates. Epilepsia 2005;46:420-30.

18. Araujo D, Santos AC, Velasco TR, et al. Volumetric evidence of bilateral damage in unilateral mesial temporal lobe epilepsy. Epilepsia 2006;47:1354-9.

19. Cook MJ, Fish DR, Shorvon SD, Straughan K, Stevens JM. Hippocampal volumetric and morphometric studies in frontal and temporal lobe epilepsy. Brain 1992;115 ( Pt 4):1001-15.

20. Thivard L, Hombrouck J, du Montcel ST, et al. Productive and perceptive language reorganization in temporal lobe epilepsy. Neuroimage 2005;24:841-51. 
21. Guye M, Le Fur Y, Confort-Gouny S, et al. Metabolic and electrophysiological alterations in subtypes of temporal lobe epilepsy: a combined proton magnetic resonance spectroscopic imaging and depth electrodes study. Epilepsia 2002;43:1197-209.

22. Didelot A, Ryvlin P, Lothe A, Merlet I, Hammers A, Mauguiere F. PET imaging of brain 5-HT1A receptors in the preoperative evaluation of temporal lobe epilepsy. Brain 2008;131:2751-64.

23. Park SA, Kim GS, Lee SK, et al. Interictal epileptiform discharges relate to 1H-MRSdetected metabolic abnormalities in mesial temporal lobe epilepsy. Epilepsia 2002;43:1385-9.

24. Bartolomei F, Khalil M, Wendling F, et al. Entorhinal cortex involvement in human mesial temporal lobe epilepsy: an electrophysiologic and volumetric study. Epilepsia 2005;46:677-87.

25. Bartolomei F, Chauvel P, Wendling F. Epileptogenicity of brain structures in human temporal lobe epilepsy: a quantified study from intracerebral EEG. Brain 2008;131:1818-30.

26. Bernasconi N, Bernasconi A, Caramanos Z, Antel SB, Andermann F, Arnold DL. Mesial temporal damage in temporal lobe epilepsy: a volumetric MRI study of the hippocampus, amygdala and parahippocampal region. Brain 2003;126:462-9.

27. Insausti R, Herrero MT, Witter MP. Entorhinal cortex of the rat: cytoarchitectonic subdivisions and the origin and distribution of cortical efferents. Hippocampus 1997;7:146-83.

28. Insausti R, Amaral DG, Cowan WM. The entorhinal cortex of the monkey: II. Cortical afferents. J Comp Neurol 1987;264:356-95.

29. Witter MP, Amaral DG. Entorhinal cortex of the monkey: V. Projections to the dentate gyrus, hippocampus, and subicular complex. J Comp Neurol 1991;307:437-59. 
30. Morrell F. Varieties of human secondary epileptogenesis. J Clin Neurophysiol 1989;6:227-75.

31. Sutula TP. Secondary epileptogenesis, kindling, and intractable epilepsy: a reappraisal from the perspective of neural plasticity. Int Rev Neurobiol 2001;45:355-86.

32. Wilder BJ. The mirror focus and secondary epileptogenesis. Int Rev Neurobiol 2001;45:435-46.

33. Schevon CA, Cappell J, Emerson R, et al. Cortical abnormalities in epilepsy revealed by local EEG synchrony. Neuroimage 2007;35:140-8.

34. Bettus G, Wendling F, Guye M, et al. Enhanced EEG functional connectivity in mesial temporal lobe epilepsy. Epilepsy Res 2008;81:58-68.

35. Wendling F, Bartolomei F, Senhadji L. Spatial analysis of intracerebral electroencephalographic signals in the time and frequency domain: identification of epileptogenic networks in partial epilepsy. Philos Transact A Math Phys Eng Sci 2009;367:297316.

36. Rosenbaum RS, Furey ML, Horwitz B, Grady CL. Altered connectivity among emotionrelated brain regions during short-term memory in Alzheimer's disease. Neurobiol Aging 2008.

37. Nadel L, Samsonovich A, Ryan L, Moscovitch M. Multiple trace theory of human memory: computational, neuroimaging, and neuropsychological results. Hippocampus 2000;10:352-68.

38. Squire LR, Stark CE, Clark RE. The medial temporal lobe. Annu Rev Neurosci 2004;27:279-306.

39. Spiers HJ, Maguire EA, Burgess N. Hippocampal amnesia. Neurocase 2001;7:357-82. 
40. Axmacher N, Schmitz DP, Weinreich I, Elger CE, Fell J. Interaction of working memory and long-term memory in the medial temporal lobe. Cereb Cortex 2008;18:2868-78.

41. Axmacher N, Mormann F, Fernandez G, Cohen MX, Elger CE, Fell J. Sustained neural activity patterns during working memory in the human medial temporal lobe. $\mathrm{J}$ Neurosci 2007;27:7807-16.

42. Wagner K, Frings L, Spreer J, et al. Differential effect of side of temporal lobe epilepsy on lateralization of hippocampal, temporolateral, and inferior frontal activation patterns during a verbal episodic memory task. Epilepsy Behav 2008;12:382-7.

43. Bosma I, Douw L, Bartolomei F, et al. Synchronized brain activity and neurocognitive function in patients with low-grade glioma: a magnetoencephalography study. Neuro Oncol 2008;10:734-44.

44. Weber B, Wellmer J, Schur S, et al. Presurgical language fMRI in patients with drugresistant epilepsy: effects of task performance. Epilepsia 2006;47:880-6.

45. Bonelli SB, Powell R, Yogarajah M, et al. Preoperative amygdala fMRI in temporal lobe epilepsy. Epilepsia 2009;50:217-27. 


\section{FIGURES LEGENDS:}

Figure 1: Differences of basal functional connectivity in controls within mesial temporal lobes depending on handedness.

All connecting lines correspond to significant basal functional connectivity. Grey lines correspond to links that are not significantly different between right- and left-handed controls. Bold lines correspond to links significantly different between right and left handed controls. Right-handed controls showed significantly higher basal functional connectivity in the left temporal lobe for the links amygdala-posterior hippocampus and anterior-posterior hippocampi (Wilcoxon rank test, corrected $\mathrm{p}<0.005$ ). Note that for the 2 groups, the link between entorhinal cortex and posterior hippocampus was not significant bilaterally.

Figure 2: Group analysis of altered basal functional connectivity prevalence in epileptogenic zone lobe (EZ) and contralateral lobe (contra). a) number of decreased links is significantly higher in the EZ compared to the contralateral hemisphere (Wilkoxon signed rank $\mathrm{p}=0,025)$. b) number of increased links is significantly higher in the contralateral temporal lobe to EZ compared to EZ temporal lobe (Wilkoxon signed rank p<0.001). 


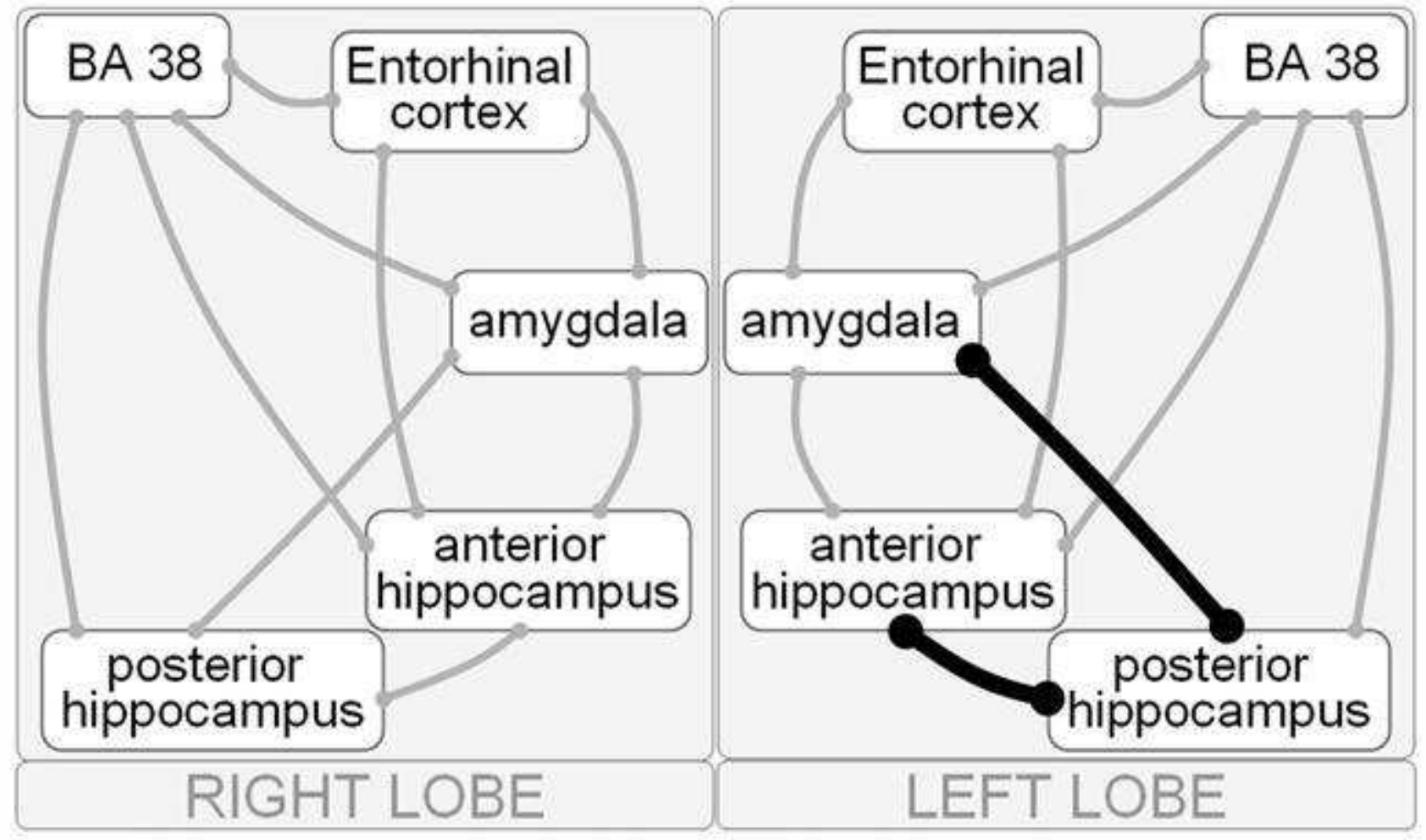

$\Longrightarrow$ Similar connectivity values in right- and left-handed control groups

- Significantly increased connectivity in right-handed compared to left-handed group 
a)

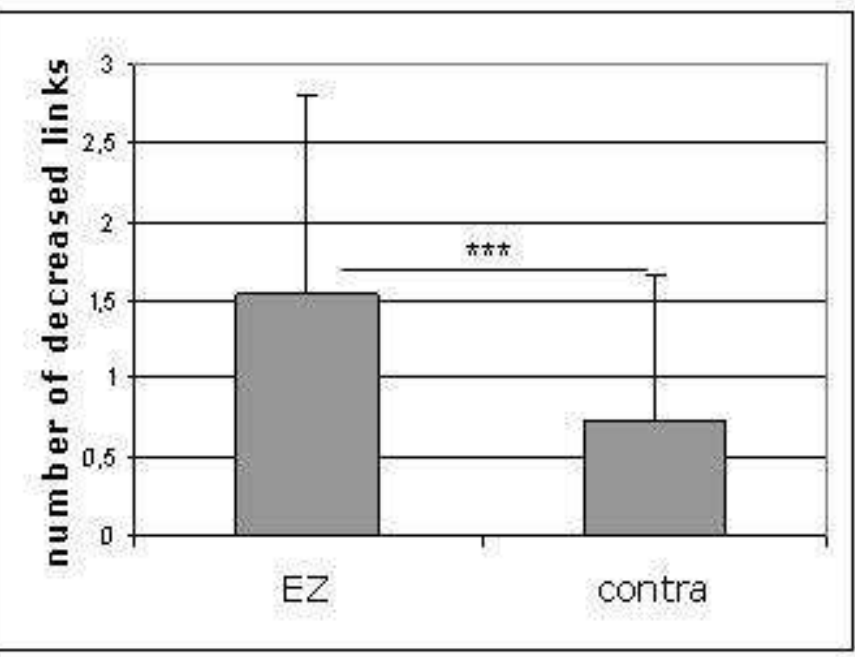

b)

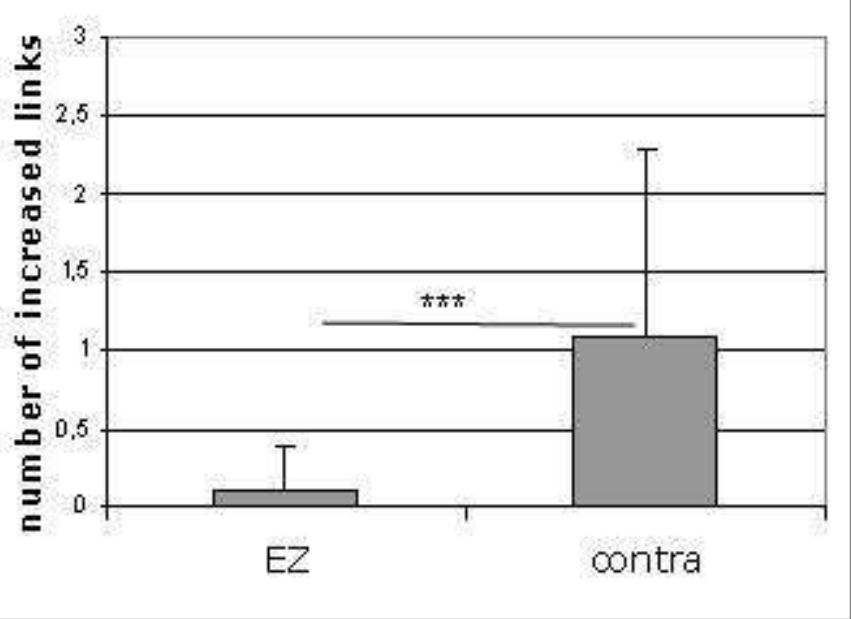

